\title{
Capecitabine Regimen
}

National Cancer Institute

\section{Source}

National Cancer Institute. Capecitabine Regimen. NCI Thesaurus. Code C160005.

A chemotherapy regimen consisting of capecitabine that may be used in the treatment

of breast (and its metastases to the central nervous system), colon, esophageal and esophagogastric junction, gastric, hepatobiliary, ovarian, fallopian tube, primary peritoneal, and rectal cancers; and pancreatic adenocarcinoma. 